\title{
Seguridad del omeprazol: ¿es adecuada la duración de los tratamientos?
}

\author{
Francisco Carranza Caricol \\ Doctor en Farmacia. Farmacéutico comunitario en Montemolín (Badajoz).
}

\section{PALABRAS CLAVE}

Omeprazol, farmacia comunitaria, abuso, dispensación

\section{ABREVIATURAS}

AINE: antiinflamatorios no esteroideos.

IBP: inhibidores de la bomba de protones. RAM: reacción adversa a medicamentos.

\section{KEYWORDS}

Omeprazole, community pharmacy, abuse, dispensation

\section{RESUMEN}

Introducción: Omeprazol es uno de los fármacos más prescritos dentro del Sistema Nacional de Salud español. Su perfil de seguridad se considera alto, aunque aparecen descritas en la ficha técnica diferentes reacciones adversas, algunas de ellas, aunque poco o muy poco frecuentes, de carácter grave. Evidencias clínicas y estudios recientes han puesto de manifiesto una relación del uso continuado de omeprazol con otras manifestaciones adversas.

Objetivos: Conocer qué parte de la población tratada sobrepasa la duración del tratamiento según lo consignado en la ficha técnica para el tratamiento de la esofagitis por reflujo y qué proporción puede presentar un riesgo potencial de sufrir alguna reacción adversa en las circunstancias en las que se han detectado.

Pacientes y métodos: Estudio observacional multicéntrico cuya recogida de datos se efectuó en 27 farmacias comunitarias ubicadas en 24 localidades de la provincia de Badajoz mediante un cuestionario estructurado con cinco preguntas relacionadas con el uso de omeprazol.

Resultados: El 60\% de los pacientes estudiados sobrepasan ampliamente el tiempo de tratamiento para tratar el reflujo gastroesofágico según la ficha técnica. El porcentaje era del 39\% en pacientes de más de 70 años. Un 4,5\% de pacientes de edad avanzada llevaba tomando omeprazol a dosis altas durante un tiempo prolongado (más de un año), circunstancia que los hace más vulnerables a sufrir alguna de las reacciones adversas descritas.

\section{Omeprazole safety: is the duration of the treatments suitable?}

\section{ABSTRACT}

Introduction: Omeprazole is one of the most prescribed drugs in the Spanish National Health System. Its safety profile is considered to be good, nevertheless there are different adverse reactions described in the summary of product characteristics, some of them, although somewhat rare or very rare, are considered serious. Clinical evidence and recent studies have shown a relationship between continued use of omeprazole and other adverse events.

Objectives: To find out how much of the treated population exceeds the duration of treatment, as stated in the Summary of Product Characteristics, for the treatment of reflux esophagitis and what proportion of this population may present potential risk of suffering any adverse reaction in circumstances that they were detected

Patients and methods: Multicenter observational study whose data collection was performed in 27 community pharmacies located in 24 towns in the province of Badajoz using a structured questionnaire with five questions related to the use of omeprazole.

Results: $60 \%$ of the patients studied amply exceed the treatment time to treat gastroesophageal reflux according to the summary of product characteristics. The 39\% was in elderly population. The $4.5 \%$ of elderly patients who taking omeprazole on high doses for a long period of time (over one year), circumstances that make them more vulnerable to suffer any adverse reactions described.

Parte de este trabajo fue presentado como comunicación en formato póster en el VI Congreso Nacional de Farmacéuticos Comunitarios celebrado en Málaga en mayo de 2014.

Recibido: 15/1/2015

Aceptado: 21/2/2015

Disponible online: 1-3-2015
Financiación: Ninguna ajena.

Conflicto de intereses: El autor declara no existir conflicto de intereses en relación con el contenido del artículo.

Cite este artículo como: Carranza F. Seguridad del omeprazol: ¿es adecuada la duración de los tratamientos?. Farmacéuticos Comunitarios. 2015 Mar 01 ; 7(1):5-9 . doi:10.5672/FC.2173-9218.(2015/Nol7).001.02

Correspondencia: Francisco Carranza Caricol (franciscocarranza@redfarma.org).

ISSN 1885-8619 @SEFAC (Sociedad Española de Farmacia Familiar y Comunitaria). Todos los derechos reservados. 


\section{Introducción}

Omeprazol es el primer fármaco comercializado de los llamados inhibidores de la bomba de protones (IBP), familia de medicamentos utilizados en el tratamiento de diversas patologías del tracto gastro-esofágico. En España es uno de los fármacos más prescritos dentro del Sistema Nacional de Salud en la actualidad (1), muy por encima del consumo en otros países del entorno europeo (2).

Las indicaciones autorizadas son diversas y la duración del tratamiento viene especificada en la ficha técnica de este medicamento (3), aunque para algunas de ellas esta duración no va expresamente definida, lo que puede dar lugar a ambigüedad en cuanto a su interpretación. Para el tratamiento de la esofagitis por reflujo, en concreto, la dosis recomendada es de $20 \mathrm{mg}$ diarios durante 4-8 semanas de tratamiento. Cuando es severa, se recomienda omeprazol a una dosis de $40 \mathrm{mg}$ una vez al día, consiguiéndose generalmente la curación en ocho semanas. En el tratamiento de mantenimiento de la esofagitis erosiva cicatrizada, se recomienda administrar $20 \mathrm{mg}$ una vez al día durante 6 a 12 meses.

Existe, por otra parte, la percepción general de que este medicamento presenta un perfil de seguridad alto (4), a pesar de que aparecen descritas en la ficha técnica diferentes reacciones adversas, algunas de ellas, aunque poco o muy poco frecuentes, de carácter grave. Tal es el caso de la hipomagnesemia $(5,6)$ en pacientes tratados durante al menos tres meses y, en la mayoría de los casos, durante un año. También riesgo de fracturas de cadera, muñeca y columna vertebral en dosis altas y durante tratamientos prolongados (más de un año), sobre todo en pacientes de edad avanzada o en presencia de otros factores de riesgo, si bien parece que existe controversia al respecto.

Sin embargo, evidencias clínicas y estudios recientes han puesto de manifiesto una relación del uso continuado de omeprazol con otras manifestaciones adversas: déficit de vitamina B12 (7), nefritis intersticial aguda $(8,9)$, candidiasis orofaríngea (10) y alucinaciones visuales (11).

El farmacéutico comunitario es testigo privilegiado del uso de este medicamento al poder constatar las dispensaciones efectuadas en pacientes, generalmente polimedicados, que reciben con prescripciones de diferentes orígenes, no solo desde la atención primaria. Y ser, además, receptores de información en cuanto al uso real de los medicamentos y sospechas de reacciones adversas relacionadas con ellos. De

\section{Sexo $\square \quad$ Edad $\square$}

1. ¿Cuánto tiempo lleva tomando omeprazol?

$$
\begin{aligned}
& \square \text { Menos de } 1 \text { año. } \\
& \text { Entre } 1 \text { y } 2 \text { años. } \\
& \text { Más de } 2 \text { años. }
\end{aligned}
$$

2. ¿Para qué se lo recetaron?

Para el reflujo gastroesofágico.

Como protector estomacal mientras toma otros medicamentos.

- Para otros problemas gástricos

3. ¿Recuerda durante cuánto tiempo se le dijo que debia tomar este medicamento? $\square \mathrm{Si}$

No

4. ¿Toma este medicamento todos los dias?

$\square \mathrm{Si}$

No

5. ¿Cuánto toma al dia?

口 $10 \mathrm{mg}$

口 $20 \mathrm{mg}$

口 $40 \mathrm{mg}$

口 $80 \mathrm{mg}$

Figura 1 Cuestionario utilizado

hecho, el gran número de dispensaciones y la continuidad de los tratamientos observados de este medicamento hace al farmacéutico comunitario formularse las siguientes preguntas: ¿se está siguiendo en la práctica clínica la duración de los tratamientos en cada una de sus indicaciones autorizadas según lo establecido en la ficha técnica? Entre los pacientes en tratamiento, ¿cuántos de ellos podrían tener mayor riesgo de sufrir las RAM conocidas?

Si bien se tiene constancia de que se han realizados estudios desde farmacias comunitarias acerca del uso de omeprazol (12), ha parecido procedente llevar a cabo el presente trabajo por cuanto afecta a un número de usuarios considerablemente mayor, de diferentes poblaciones y en los que se consideran las diferentes dosis que puedan estar administrándose.

Se persigue como objetivo de este trabajo conocer: $a$ ) qué parte de la población tratada sobrepasa la duración del tratamiento según lo consignado en la ficha técnica para el tratamiento de la esofagitis por reflujo, y b) qué proporción de la misma puede presentar un riesgo potencial de sufrir alguna reacción adversa en las circunstancias en las que se han detectado en ensayos y estudios clínicos. Como objetivo secundario se pretendió saber cuántos pacientes de la población estudiada recuerdan la duración del tratamiento según les había indicado el médico.

\section{Pacientes y métodos}

Estudio observacional descriptivo transversal multicéntrico cuya recogida de datos se efectuó en 27 farmacias comunitarias ubicadas en 24 localidades de la provincia de Badajoz. Se incluyeron en el estudio todos aquellos pacientes o sus cuidadores que durante el periodo comprendido entre el 15 de enero y el 15 de marzo de 2014, acudieron a la farmacia con una prescripción de omeprazol en cualquiera de sus presentaciones de $10 \mathrm{mg}, 20 \mathrm{mg}$ o $40 \mathrm{mg}$, que aceptaron participar y que estaban capacitados para entender las preguntas del cuestionario. Quedaron excluidas aquellas personas que retiraron la medicación que no eran ni el paciente destinatario ni su cuidador.

Se elaboró un cuestionario estructurado con cinco preguntas relacionadas con el uso de omeprazol, además de las correspondientes a sexo y edad (figura 1). Se pilotó en la farmacia 


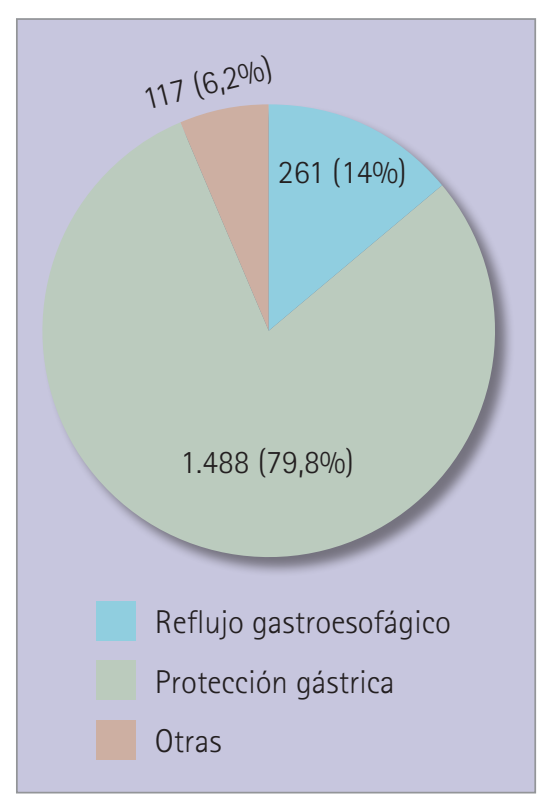

Figura 2 Distribución por indicaciones

del autor antes de iniciar el estudio en las farmacias participantes. Los cuestionarios fueron cumplimentados por los pacientes (o cuidadores) que cumplían el criterio de inclusión, orientados, en los casos que se requería, por los farmacéuticos. Se pretendía saber de los usuarios cuánto tiempo llevaban tomando este medicamento, si sabían para qué lo usaban, si recordaban cuánto tiempo se les indicó que debían estar tomándolo, si la administración era diaria y en qué dosis. Se consideró que los pacientes ancianos que tomaban una dosis de $40 \mathrm{mg}$ de omeprazol durante más de 1 año estaban en riesgo de sufrir una RAM.

Como variable de estudio se utilizó el número de pacientes afectados por el uso inadecuado del medicamento, categorizado en función de las distintas respuestas a las preguntas del cuestionario. Los resultados obtenidos se procesaron en una base de datos tipo Access ${ }^{\circledR}$ de Microsoft Office ${ }^{\circledR}$ diseñada al efecto.

\section{Resultados}

El número total de pacientes participantes en el estudio fue de 1.866 .

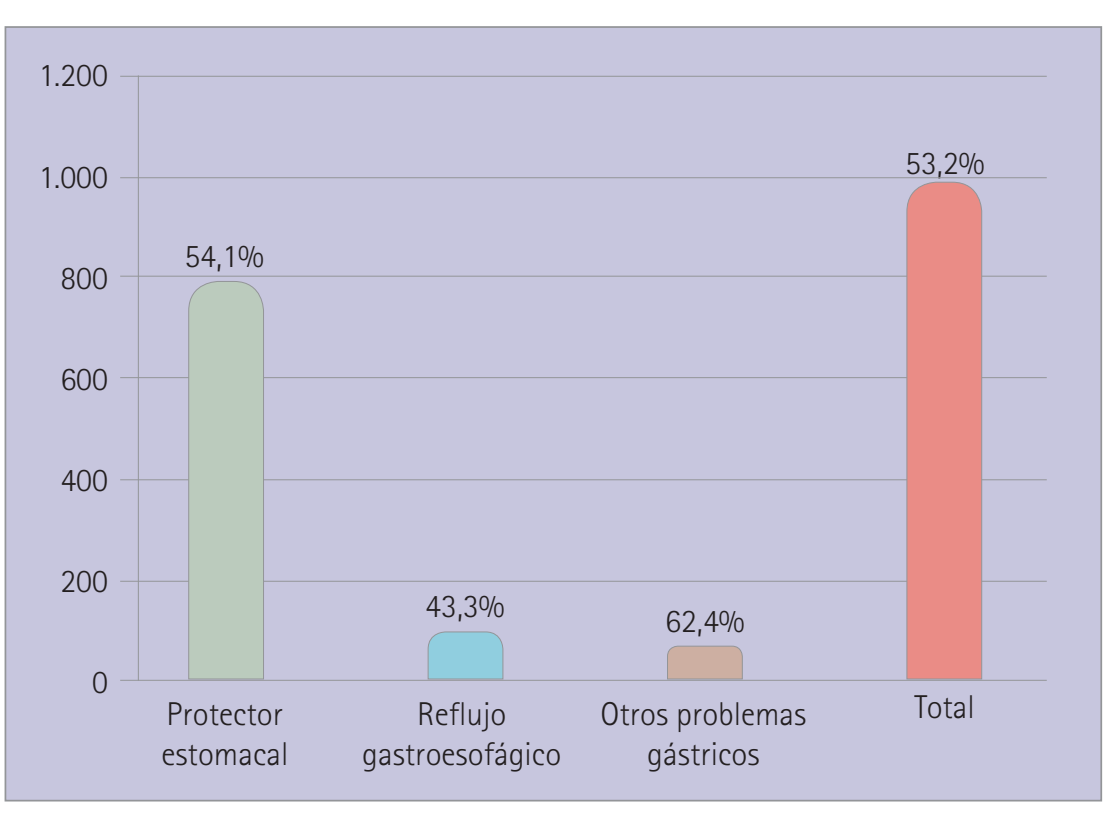

Figura 3 Número de pacientes que no recuerdan la duración del tratamiento der el cuestionario. Las indicaciones para las cuales toman omeprazol los pacientes se muestran en la figura 2.

De los pacientes que toman omeprazol para tratar problemas de reflujo gastroesofágico, 157 (60,1\%) llevan más de 1 año con este tratamiento, y de estos $149(57,1 \%)$ lo toman a diario frente a $112(42,9 \%)$ que lo hacen cuando lo necesitan (tabla 1).

Considerando "de edad avanzada" a los mayores de 70 años, del total de pacientes consultados que usan este medicamento, 765 (41,0\%) igualaban o superaban esta edad.

Con respecto al tiempo y la continuidad de tratamiento, 722 (38,7\%) llevaban más de un año de tratamiento, de los cuales, 645 (34,6\%) estaban tomando el medicamento ininterrumpidamente a diario. De ellos, 84 (4,5\%) estaban tomando $40 \mathrm{mg}$ o más.

Del total de pacientes incluidos en el estudio, $873(46,8 \%)$ manifestaron recordar de las indicaciones de su médico el tiempo que debían mantener su tratamiento. La distribución de los pacientes que no recuerdan la duración del tratamiento se muestra en la figura 3.
No hubo ninguna negativa a respon-

Tabla 1 Pacientes que toman omeprazol para tratar el reflujo gastroesofágico

\begin{tabular}{l|c|c|c|} 
& $\begin{array}{c}\text { Menos de un año } \\
\text { de tratamiento }\end{array}$ & $\begin{array}{c}\text { Más de un año } \\
\text { de tratamiento }\end{array}$ & Total \\
\hline Lo toman a diario & 75 & 74 & 149 \\
No lo toman a diario & 29 & 83 & 112 \\
Total & 104 & 157 & 261 \\
\hline
\end{tabular}

\section{Discusión}

Aunque fuera de los objetivos de este estudio, parece importante traer a esta discusión el dato hallado sobre el elevado número de pacientes que usan omeprazol como protector estomacal cuando toman otros medicamentos, circunstancia que concuerda con lo publicado en recientes estudios (13-15) que lo achacan a una incorrecta utilización de la mal llamada "protección gástrica”, ampliada de forma indiscriminada para proteger cualquier molestia dispéptica producida por medicamentos, a pesar de que no hay pruebas de la eficacia ni de la seguridad de esta actuación terapéutica (16). Si bien aparece en ficha técnica el uso de omeprazol para la prevención de ulcus gástrico y duodenal asociados a antiinflamatorios no esteroideos (AINE) en pacientes de riesgo, diariamente se constata desde la farmacia comunitaria multitud de prescripciones de este medicamento como protector gástrico de forma generalizada, en pacientes polimedicados, estén incluidos o no AINE en sus tratamientos. Por otra parte, ha de considerarse que el consumo de AINE no parece ser responsable directamente de este elevado uso de omeprazol puesto que aunque el de AINE ha sufrido incrementos paralelos en los primeros años de la década de los 2000 , se ha producido cierta tendencia a disminuir a partir de 2009, tendencia contraria a la evolución de las dispensaciones de omeprazol (17).

Como se ha señalado, existen estudios similares al presente, si bien 
en este caso se ha pretendido ampliar considerablemente el tamaño de la muestra (1.866 encuestas) y distribuir la muestra de forma multicéntrica, de manera que se han incluido farmacias del medio rural y del medio urbano, en diferentes rangos poblacionales. Se prefirió centrar el análisis solamente en el uso de omeprazol y obviar el resto de IBP actualmente autorizados, poniendo particular interés en conocer la dosis diaria indicada para estos pacientes a fin de indagar qué parte de la muestra estudiada podría considerarse que utiliza dosis altas en su tratamiento, vinculando este hecho a la posibilidad de relacionarlo con la aparición de determinadas reacciones adversas ligada precisamente a la administración de dosis altas.

En relación al primer objetivo planteado, sorprende el elevado porcentaje de pacientes (en torno al 60\%) que supera el año de tratamiento con omeprazol cuando se les ha prescrito para tratar problemas de reflujo gastroesofágico. La ficha técnica de este fármaco indica un periodo de 6 a 12 meses para el caso de esofagitis erosiva cicatrizada, que requiere mayor tiempo de tratamiento. Este hecho plantea varias interrogantes acerca de la duración óptima de este tratamiento, como que, tal vez, los plazos indicados en la ficha técnica no sean lo suficientemente adecuados para lograr la curación completa de esta patología; quizás la evidencia clínica disponible hable en favor de tratamientos más prolongados. Pero también pudiera ser que se esté produciendo cierto relajamiento a la hora de hacer seguimiento de estos tratamientos. En este punto, el farmacéutico tendría algo o mucho que decir y que hacer. Es de notar, además, que se observa un hecho que no debía ocurrir: existen pacientes que no recuerdan hasta cuando han de seguir tomando este tratamiento; en este estudio, un 43,3\% de los pacientes consultados. Sería, por tanto, el momento de activar protocolos ya consensuados sobre dispensación y seguimiento farmacoterapéutico en la farmacia comunitaria para corregir esta anomalía.

Por lo que respecta al segundo de los objetivos perseguidos, éste relacionado con la seguridad del omeprazol, es preciso recordar antes que han sido detectadas nuevas reacciones adversas para este fármaco, probablemente como consecuencia de su uso tan elevado y extendido. En general, estas reacciones adversas aparecen relacionadas con el uso prolongado de este fármaco, con la edad avanzada del paciente, con la continuidad del tratamiento y con el uso de dosis altas (16). En este sentido se ha encontrado un dato cuando menos preocupante: un 4,5\% de los consultados son pacientes de edad avanzada, con tratamientos ininterrumpidos superiores a un año y que usan dosis altas. Es decir, que existe de hecho entre los pacientes que atendemos en nuestras farmacias una población muy vulnerable, en la que confluyen varios factores de riesgo a la vez para sufrir reacciones adversas, sobre la que se hace perentorio estar en permanente vigilancia y actuar si se diera el caso. Así pues, cabe la necesidad de ampliar los procedimientos sugeridos más atrás a intervenciones de farmacovigilancia, notificaciones en tarjeta amarilla incluidas, que garanticen una correcta, pero sobre todo efectiva, atención farmacéutica en nuestras farmacias.

Se había propuesto también, pero como objetivo secundario en este estudio, conocer el número de pacientes en la población estudiada que recuerda la duración del tratamiento según les había indicado el médico. En concreto, la pregunta que se les formuló fue: ¿recuerda durante cuánto tiempo se le dijo que debía tomar este medicamento? Nuevamente se encuentra un dato inquietante: más de la mitad de los pacientes entrevistados dicen no recordar, bien porque lo hayan olvidado, bien porque el médico hubiera omitido esta información, el tiempo que deberían estar tomando omeprazol. Otra vez se abre aquí un campo de actuación del farmacéutico comunitario que no debería dejar pasar en modo alguno vistos los resultados obtenidos en este estudio. Es seguro que corregir esta anomalía en la práctica diaria, informando, derivando el paciente al médico o ambas actuaciones a la vez, llevaría a cero esta cifra $y$, por consiguiente, se habría conseguido el objetivo fundamental de la atención farmacéutica que no es otro que conseguir resultados que mejoren la calidad de vida del paciente en relación al uso de los medicamentos (18).

\section{Conclusiones}

El 60\% los pacientes encuestados sobrepasa ampliamente el tiempo de tratamiento con omeprazol para tratar el reflujo gastroesofágico según indica la ficha técnica de este fármaco.

El 4,5\%, de la muestra, de edad avanzada lleva tomando omeprazol a dosis altas durante un tiempo prolongado (más de un año), circunstancias que la hace más vulnerable a sufrir alguna de las reacciones adversas descritas.

Más de la mitad de los pacientes consultados $(53,2 \%)$ no recuerda las indicaciones de su médico en cuanto a la duración de su tratamiento con omeprazol.

\section{Agradecimientos}

Quiero hacer constar mi más sincero agradecimiento a los compañeros que han contribuido en la obtención de datos sin cuya colaboración entusiasta no habría sido posible la realización de este trabajo: M. Pulgarín y E. Tevar (Azuaga), M. L. Reynolds (Entrín Bajo), J. M. Franco (Helechosa de los Montes), Lydia Díaz (Rena), M. P. Fuentes (Oliva de la Frontera), Fernando Fernández (Calzadilla de los Barros), J. Fernández de Alarcón (Almendralejo), E. de Tena (Mérida), J. Álvarez (Calera de León), M. J. Ramírez (Valencia de las Torres), E. Comerón (Táliga), A. Jiménez (Puebla del Prior), A. Fernández (Puebla de Sancho Pérez), F. Venegas (Usagre), C. González Escalada (La Albuera), J. M. Fortis (Puebla de la Calzada), P. Durán (Villafranca de los Barros), L. E. García (Calamonte), F. J. García-Moreno (Valverde de Leganés), M. V. Fernández (Balboa), L. González (Pallares) y J. Palo, J. M. Noa, C. Gervasini, J. Martínez de la Concha (Badajoz).

\section{Referencias bibliográficas}

1. AEMPS. Utilización de medicamentos antiulcerosos en España durante el periodo 2000-2012 [Internet]. [Consultado 12/10/2014]. Disponible en: http://www.aemps.gob.es/medicamentosUsoHumano/observatorio/docs/antiulcerosos.pdf

2. García del Pozo J. Estudio de utilización de antiulcerosos en España (2000-2008). Información Terapéutica del Sistema Nacional de Salud. 2009; 33(2): 49-54.

3. AEMPS. Ficha técnica omeprazol [Internet]. [Consultado 12/10/2014]. 
Disponible en: http://www.aemps. gob.es/cima/pdfs/es/ft/63230/ FT_63230.pdf

4. Esplugues JV, Martí-Cabrera M, Ponce J. Seguridad de la utilización de los inhibidores de la bomba de protones. Medicina Clínica. 2006; 127: 790795. doi:10.1157/13095825

5. Gato Diez A, Córdoba Soriano JG, Mora Escudero I. Hipomagnesemia grave debido al tratamiento prolongado con omeprazol. Medicina Clínica. 2011 ; 136: 84-85. doi:10.1016/j. medcli.2009.10.019

6. Cano Megías M, Álvarez Santirso R, Iglesias Lozano P, Carrasco de la Fuente M, Pérez López G. Hipomagnesemia relacionada con el uso de inhibidores de la bomba de protones, diarrea e intolerancia a la lactosa. Endocrinol Nutr. 2011; 58: 550-554. doi:10.1016/j.endonu.2011.03.007

7. Lam JR, Schneider JL, Zhao W, Corley DA, Proton Pump Inhibitor and Histamine 2 Receptor Antagonist Use and Vitamin B12 Deficiency. JAMA. 2013; 310(22): 2435-2442. doi:10.1001/jama.2013.280490

8. Borruel Aguilar MJ, Brun Izquierdo A, Marco Catalán P, Morte Pascual A.
Nefritis intersticial aguda por omeprazol. A propósito de un caso. Semergen. 2007; 33: 318-9. doi:10.1016/ S1138-3593(07)73904-7

9. Vera Rivera M, Pou Potau M, Botey Puig A. Nefritis intersticial por omeprazol: una causa reversible de insuficiencia renal aguda. Medicina Clínica. 2002; 118: 139. doi:10.1016/S00257753(02)72274-4

10. Pérez Prim FJ, Vila I. Tratamiento continuado con omeprazol y aparición de candidiasis orofaringea. Aten Primaria. 2002; 30: 663-664. doi:10.1016/S0212-6567(02)79132-4

11. López Serrano B, Frías Vargas M, Medina Cuenca P, Cortés Durán PM. Alucinaciones visuales secundarias a tratamiento continuado con omeprazol. A propósito de un caso. Semergén. 2009; 35(09): 475-477. doi:10.1016/ S1138-3593(09)72848-5

12. Ezquieta MF, Igoa R. Características de las dispensaciones de omeprazol en una farmacia comunitaria. Farmacéuticos Comunitarios 2010; 2(2): 51-55.

13. De Burgos Lunar C, Novo de Castillo S, Llorente Díaz E, Salinero Fuerte MA. Estudio de prescripción-indicación de inhibidores de la bomba de protones.
Revista Clínica Española 2006; 206(6): 266-270. doi:10.1157/13088585

14. San José AY, Velarde Mayol C, Gómez de Caso Canto YA. Variabilidad de la práctica clínica: calidad de prescripción de los inhibidores de la bomba de protones. Revista de Calidad Asistencial. 2012; 27(3): 182-183. doi:10.1016/j.cali.2012.01.009

15. Hermida Ameijerira A, Cabana González B, Lorenzo Zúñiga V. Prevalencia de prescripción-indicación de protectores gástricos en pacientes hospitalizados. Gaceta Sanitaria. 2007; 21(5): 412-415. doi:10.1157/13110449

16. Madrigalejos R. Inhibidores de la bomba de protones y el balance riesgo/beneficio. Butlletí d'informació terapéutica, 2013; 24(2): 7-10.

17. Simo Miñana J, Gorricho Mendívil J. Consumo creciente y en paralelo de antiulcerosos y antinflamatorios en España. Formación Médica Continuada en Atención Primaria, 2012; 19(7): 453. doi:10.1016/S11342072(12)70435-8

18. Ministerio de Sanidad y Consumo. Consenso sobre Atención Farmacéutica, 2001. Ars Pharmaceutica. 2001; 42(3-4); 221-241. 